\title{
Photometric and spectroscopic variability of the B5Ille star HD 171219
}

\author{
Laerte Andrade $^{1, \star}$, Eduardo Janot-Pacheco ${ }^{2}$, Marcelo Emilio ${ }^{1}$, and Yves Frémat ${ }^{3}$ \\ ${ }^{1}$ Universidade Estadual de Ponta Grossa, Av. Carlos Cavalcanti, 4748, 84030-900, Ponta Grossa, Brazil \\ ${ }^{2}$ Universidade de São Paulo/IAG, rua do Matão, 1226 - Cidade Universitária, 05508-900, São Paulo, Brazil \\ ${ }^{3}$ Royal Observatory of Belgium, 3 avenue circulaire, B1180 Brussels, Belgium
}

\begin{abstract}
We analyzed the Be star HD 171219, observed with the CoRoT satellite during a 77.56 day run, in order to determine its physical and pulsation characteristics. Highresolution ground-based spectroscopic observations with HARPS and SOPHIE were also obtained during the month preceding the CoRoT observations. Twenty frequencies have been detected in the object, some of them compatible with non-radial g-modes, including a quintuplet centered around $1.113 \mathrm{c} / \mathrm{d}(12.88 \mu \mathrm{Hz})$ identified as a pulsation of degree $\ell \sim 2$. The star underwent at least 6 small outbursts during the CoRoT observations. The relative intensity of the main frequencies varied after each outburst suggesting that the stability of the star and the feeding of the envelope are linked to the pulsation regime ([1]).
\end{abstract}

\section{Photometric analysis of CoRoT data}

The Be Star HD 171219 was continually observed by the CoRoT satellite during 77.56 days from July to September 2010, with a cadence of 32 seconds. Its light curve (Fig. 1a) contains 209,418 photometric observations. Close examination shows the occurrence of a medium-size outburst around CJD 3876 and four minor outbursts close to CJD = 3847, 3853, 3894 and 3905.

We applied the CLEANEST algorithm ([2,3]) to look for pulsation frequencies (Fig. 1b). We have twenty periodicities in the light curve, some of them compatible with a g-mode quintuplet $\left(f_{1}=\right.$ $1.072 \mathrm{c} \mathrm{d}^{-1}, f_{2}=1.089 \mathrm{~cd}^{-1}, f_{3}=1.113 \mathrm{c} \mathrm{d}^{-1}, f_{4}=1.130 \mathrm{c} \mathrm{d}^{-1}$ and $\left.f_{5}=1.146 \mathrm{c} \mathrm{d}^{-1}\right)$.

\section{Ground-based spectroscopic analysis}

High-resolution spectra were obtained during a 20-day period with two echelle spectrographs, HARPS and SOPHIE. With 53 good quality spectra available, we rederived and improved the stellar parameters by directly comparing $2 \mathrm{D}$ spectra that account for first order rapid rotation effects (stellar flattening and gravitational darkening) to observations made of a combination of the best available spectra ([4]).

We also applied the CLEANEST method to the time series of the spectroscopic data. Because of the scarcity of spectra, there was less information on non-radial pulsations from line profile variations to be extracted ([5]), but peaks around the multiplet frequencies and their harmonics appear clearly.

\footnotetext{
^laerteandrade@uepg.br
} 


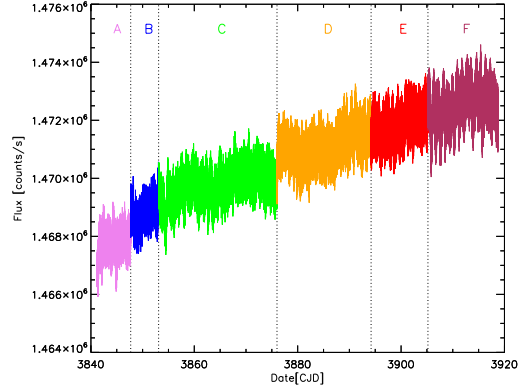

(a) CoRoT light curve of HD 171219. Colored regions (A) through (F) are divided by the ocurrences of minor or medium outbursts.

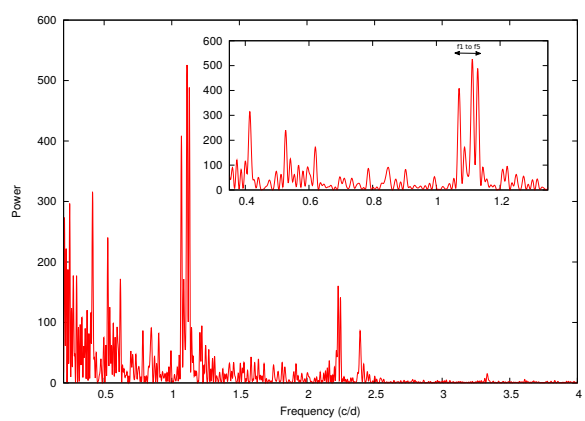

(b) Power spectrum from light curve on the left. Inset: Zoom of the region containing the g-mode $\ell \sim 2$ quintuplet $\left(f_{1}\right.$ to $f_{5}$ ) centered around $1.113 \mathrm{c} / \mathrm{d}$.

Figure 1: Photometric analysis of CoRoT data.
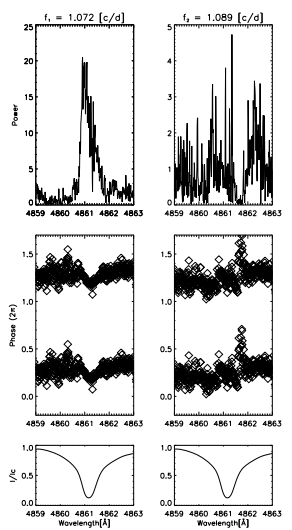
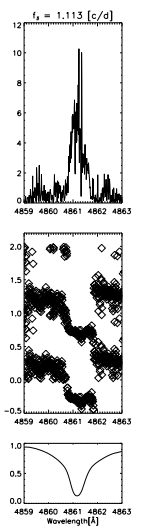
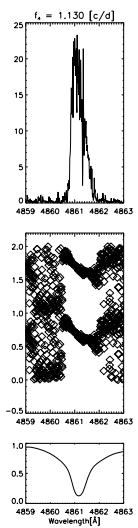
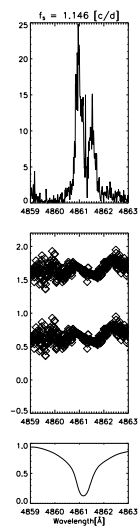

Figure 2. Power (top panel) and phase (middle panel) variation of quintuplet frequencies along the mean HARPS and SOPHIE $\mathrm{H} \beta$ line profile (bottom panel).

In Figure 2 we plotted the amplitudes and phases of the variations of the multiplet frequencies as a function of the position in the $\mathrm{H} \beta$ Balmer line profile, calculated by CLEANEST along the mean SOPHIE + HARPS observations. Following [6] we estimated the value of the NRP degree $\ell=2 \pm 1$.

Acknowledgments: Work supported in Brazil by FAPESP, CNPq and CAPES (PNPD). Partially based on observations made with the 3.6-m telescope at La Silla Observatory under the ESO Large Programme LP185.D-0056.

\section{References}

[1] Andrade, L., Janot-Pacheco, E., Emilio, M., et al., A\&A, 603, A41 (2017)

[2] Emilio, M., Andrade, L., Janot-Pacheco, E., et al., A\&A, 522, A43 (2010)

[3] Foster, G., ApJ, 109, 1889 (1995)

[4] Frémat, Y., Zorec, J., Hubert, A.-M., et al., A\&A, 440, 305 (2005)

[5] Neiner, C., Floquet, M., Samadi, R., et al., A\&A, 546, 47 (2012)

[6] Telting, J. H., \& Schrijvers, C., A\&A, 317, 723 (1997) 